\title{
Systemic Treatment and Surgery versus Systemic Treatment Alone for Metastatic Breast Cancer
}

\author{
Glauce Romeiro de Almeida ${ }^{1}$ \\ (iD) Antonio Silvinato ${ }^{1}$ \\ Wanderley Marques Bernardo² \\ Created on: 22 May, 2020 \\ 1. Programa Diretrizes da Associação Médica Brasileira, São Paulo, SP, Brasil \\ 2. Coordenador do Programa Diretrizes da Associação Médica Brasileira, São Paulo, SP, Brasil \\ E-mail: wmbernardo@usp.br
}

http://dx.doi.org/10.1590/1806-9282.66.6.710

The Guidelines Project, an initiative of the Brazilian Medical Association, aims to combine information from the medical field in order to standardize producers to assist the reasoning and decision-making of doctors.

The information provided through this project must be assessed and criticized by the physician responsible for the conduct that will be adopted, depending on the conditions and the clinical status of each patient.

\section{INTRODUCTION}

Breast cancer is the leading cause of cancer-related mortality among women worldwide ${ }^{(1)}$. In Brasil, and according to the National Institute of Cancer (INCA), breast cancer is also the type of cancer that mostly affects women in the country (except for non-melanoma skin cancer). For 2019, there were an estimated 59,700 new cases, which represents an incidence rate of 51.29 cases per 100,000 women ${ }^{(2)}$.

From all new cases of breast cancer diagnosed worldwide every year, approximately $60 \%$ to $65 \%$ are hormone receptor (HR) positive, $20 \%$ to $25 \%$ are human epidermal growth factor receptor 2 (HER2) positive, and $15 \%$ to $18 \%$ are triple-negative (estrogen receptor-negative, progesterone receptor-negative, HER2-negative). The expression of these biological markers is correlated with the prognosis and response to treatment and, therefore, plays an important role in treatment decisions ${ }^{(3)}$.
The prognosis of a patient is determined by the anatomic extension and pathobiological characteristics of their cancer, established during the staging ${ }^{(4)}$. As for metastatic breast cancer, in its initial presentation, the mean survival remains at around 18 to 24 months, and this variation may be extended to many years. This group of patients is usually treated with palliative intent; therefore, surgery is performed for the relief of symptoms. However, this approach was defined before modern advances in systemic treatments and supportive care ${ }^{(5.6)}$.

Mammography screening and other exams with improved technologies have resulted in fewer patients with inoperable presentations, and the introduction of new systemic treatments and targeted therapies, in particular, has brought a significant improvement in survival among patients with metastatic breast cancer over the past decade ${ }^{(5)}$. Having said this, it is important 
to emphasize that the role of surgery in the context of metastatic breast cancer, in its initial presentation, remains controversial ${ }^{(5,6)}$.

Some researchers have postulated that the physiological stress from surgery under general anesthesia promotes metastatic proliferation. In addition, the primary tumor was thought to inhibit angiogenesis in metastatic lesions. However, these theories against the surgical resection of the primary tumor in the context of metastatic breast cancer were based on studies with animals, without translational clinical parameters to determine to what extent they affected survival(6.7).

Currently, the common practice is still to reserve the surgical resection of the primary metastatic tumor to patients with bleeding, ulceration, or resistant pain. In this context, the evidence of survival benefits from surgery of the primary tumor has been conflicting $^{(8,9,10,11)}$.

\section{OBJECTIVE}

The objective of this assessment is to identify the benefits and harms of systemic therapy with surgery in the treatment of patients with metastatic breast cancer, compared with systemic therapy alone.

\section{METHODS}

The clinical question is: What is the impact of systemic therapy with surgery in the treatment of patients with metastatic breast cancer on overall mortality outcomes (death from any cause) and quality of life, compared to systemic therapy alone?

The eligibility criteria for the studies are:

- Adult patients with metastatic breast cancer;

- Treatment by systemic therapy with surgery compared with systemic therapy alone;

- Outcomes - death (any cause); recurrence and quality of life;

- Excluded intermediate outcomes;

- Randomized clinical trial;

- No time or language restrictions;

- Full text available for access.

The search for evidence will be conducted on the virtual database Medline using the following search strategy -(Breast Neoplasm OR Breast Neoplasms OR Breast Tumor OR Breast Tumors OR Breast Cancer OR Breast Carcinoma OR Breast Carcinomas) AND (stage IV OR metastatic) AND (primary surgery OR Primary Tumor Resection OR Primary Tumor Surgery OR primary site treatment OR surgical resection) AND Random*; and on CENTRAL / Cochrane with the search strategy - (breast cancer) AND (stage IV OR metastatic) AND (primary surgery OR surgery OR resection). The search on these databases was performed by April 2020, along with a systematic review as recommended by the Preferred Reporting Items for Systematic Reviews and Meta-Analyses (PRISM) ${ }^{(12)}$.

We will extract the following data from the studies: name of the author and year of publication, study population, intervention and comparison methods, the absolute number of deaths and adverse events (if any), variations in the quality of life between the groups, and follow-up time.

Randomized clinical trials will have their risk of biases analyzed according to the following criteria: randomization, blinded allocation, double-blinding, losses, prognostic characteristics, presence of relevant outcome, time for the outcome, the method for outcome measurement, sample size calculation, early interruption, presence of other biases.

The results will be expressed by the difference of the mean (MD) or SMD for continuous results. We will use hazard ratio (HR) for the results of time for the event and difference in risk for dichotomous outcomes. The confidence level adopted was $95 \%$.

The results of the studies included will be meta-analyzed by RevMan $5.3^{(13)}$, and HR will be the final measure used to support the synthesis of evidence that will answer the clinical question (survival analysis) of this review. We will use the inverse variance method to estimate the size of the combined effect for the results and the random-effects model by default, since we expect clinical or methodological heterogeneity, or both, in the studies included.

The heterogeneity was inspected graphically using forest plots that exhibit the effects of individual studies with confidence intervals (CIs) of 95\%. When appropriate, we will evaluate the heterogeneity among the studies using the $\mathrm{Chi}^{2}$ statistics (considering a value of $\mathrm{p}<0.10$ as significant). We will also use $\mathrm{I}^{2}$ statistics as an approximate guide to interpret the magnitude of heterogeneity: a value of $\mathrm{R}^{2}$ between $30 \%$ and $60 \%$ is indicative of moderate heterogeneity, while values greater than $50 \%$ are considered substantial heterogeneity ${ }^{(14)}$.

The quality of evidence will be graded as high, moderate, low, or very low using the GRADE (Grades 
of Recommendation, Assessment, Development, and Evaluation) ${ }^{(15)}$ instrument, taking into account the risk of bias, the presence of inconsistency, vagueness or indirect evidence in the meta-analysis of the outcomes of death and adverse events, and the presence of publication bias.

\section{RESULTS}

The search for evidence retrieved 1044 papers, of which 3 (RCTs) were selected based on their title and abstract $^{(16-18)}$ on systemic treatment with surgery in patients with metastatic breast cancer, in comparison with systemic treatment alone. The 3 studies that met the eligibility criteria were then were accessed for analysis of their full text. The 3 studies were selected to support this assessment; the grounds for exclusion are available in the references, Figure 1 under ANNEXES.

The population included comprises 714 metastatic breast cancer patients who underwent breast surgery associated to systemic treatment $(\mathrm{N}=356)$ compared with systemic therapy alone $(\mathrm{N}=358)$, and followed-up to measure the outcomes of death, recurrence, and quality of life for an average of 23 to 40 months (Table 1).

Regarding the risks of bias in the 3 studies included ${ }^{(16-18)}$, all had differences in prognostic characteristics between the intervention and control groups that could interfere with the results obtained (e.g. number of women aged less than 55 years, SR-positive and HER2-negative tumors, cT3, single bone metastases), and one study presented early interruption (5 years) due to poor recruitment; thus, the overall risk of the studies can be considered moderate (Table 2).

TABLE 1. CHARACTERISTICS OF THE STUDIES INCLUDED

\begin{tabular}{|c|c|c|c|c|c|}
\hline Study & $\begin{array}{l}\text { Population } \\
\text { (N) }\end{array}$ & $\begin{array}{l}\text { Intervention } \\
(\mathrm{N})\end{array}$ & $\begin{array}{l}\text { Comparison } \\
\text { (N) }\end{array}$ & Outcomes & $\begin{array}{l}\text { Time (medi- } \\
\text { an, months) }\end{array}$ \\
\hline $\begin{array}{l}\text { Badwe, } \\
2015\end{array}$ & $\begin{array}{l}(\mathrm{N}=350) \\
\text { Women (mean age of } 48 \\
\text { years) in India, with de } \\
\text { novo metastatic breast } \\
\text { cancer stage IV and } \\
\text { response to initial che- } \\
\text { motherapy ( } 96 \% \text { ) or who } \\
\text { received initial endocrine } \\
\text { therapy (4\%). } \\
\text { Only } 9 \text { of } 107 \text { HER2+ } \\
\text { received targeted therapy. }\end{array}$ & $\begin{array}{l}\text { ( } \mathrm{N}=173 \text { ) } \\
\text { Mastectomy or conservative breast surgery with } \\
\text { complete dissection of the axillary lymph nodes } \\
\text { (locoregional treatment), followed by standard } \\
\text { postoperative treatment of RT in the thoracic } \\
\text { wall or remaining breast. Pre-menopausal } \\
\text { women with menstruation after chemothera- } \\
\text { py had ovarian ablation (not received by } 22 \% \\
\text { in this group and 33\% in the control group). } \\
\text { Hormone receptors positive tumors received } \\
\text { endocrine therapy after locoregional treatment } \\
\text { or initial chemotherapy until their progression. }\end{array}$ & $\begin{array}{l}(\mathrm{N}=177) \\
\text { ST } \\
\text { without } \\
\text { locoregional } \\
\text { treatment }\end{array}$ & $\begin{array}{l}\text { Overall surviv- } \\
\text { al (OS) } \\
\text { Local pro- } \\
\text { gression-free } \\
\text { survival (local } \\
\text { PFS) } \\
\text { Distant pro- } \\
\text { gression-free } \\
\text { survival (dis- } \\
\text { tant PFS) }\end{array}$ & $\begin{array}{l}\text { Mean of } 23 \\
\text { months }\end{array}$ \\
\hline Soran, 2016 & $\begin{array}{l}\text { (N=274) } \\
\text { Women in Turkey with de } \\
\text { novo breast cancer stage } \\
\text { IV previously untreated. } \\
\text { Targeted therapy for all } \\
\text { HER2+ }\end{array}$ & $\begin{array}{l}\text { (N=138) } \\
\text { Mastectomy or conservative breast surgery with } \\
\text { tumor-free margins. } \\
\text { SLN biopsy for patients with clinically negative } \\
\text { lymph nodes. } \\
\text { Axillary drainage levels I and II required for } \\
\text { SLN-positive patients, patients with clinically } \\
\text { positive lymph nodes, and SNL not identified } \\
\text { during surgery. } \\
\text { All women treated with conservative breast sur- } \\
\text { gery underwent whole-breast RT 3-6 months } \\
\text { after surgery; RT for the breast, local lymph } \\
\text { node chains, chest wall, and metastatic site by } \\
\text { choice of the doctor. }\end{array}$ & $\begin{array}{l}(\mathrm{N}=136) \\
\text { ST } \\
\text { without } \\
\text { locoregional } \\
\text { treatment }\end{array}$ & $\begin{array}{l}\text { OS } \\
\text { Local PFS }\end{array}$ & $\begin{array}{l}\text { Mean of } 40 \\
\text { months }\end{array}$ \\
\hline $\begin{array}{l}\text { Fitzal, } 2018 \\
\text { Prematurely } \\
\text { interrupted } \\
\text { after } 5 \text { years } \\
\text { due to poor } \\
\text { recruitment. }\end{array}$ & $\begin{array}{l}(\mathrm{N}=90) \\
\text { Women in Austria with } \\
\text { breast cancer stage IV } \\
\text { previously untreated. } \\
\text { Targeted therapy applied } \\
\text { in } \\
\text { HER2 + }\end{array}$ & $\begin{array}{l}\text { (N=45) } \\
\text { Lumpectomy or mastectomy without tumors } \\
\text { in the margins, in addition to axillary dissection } \\
\text { level I and II or sentinel lymph node biopsy. } \\
\text { RT performed at the discretion of the research- } \\
\text { er, initiated within } 6 \text { months after the surgery, } \\
\text { but not concurrent to chemotherapy } \\
\text { ST included chemotherapy, anti-HER2 therapy, } \\
\text { or anti-hormonal therapy, at the discretion of } \\
\text { the investigator. } \\
\text { T3 cancer in } 22 \% \text {. }\end{array}$ & $\begin{array}{l}\text { (N = 45) } \\
\text { ST } \\
\text { In this group } \\
\text { without } \\
\text { surgery, local } \\
\text { surgery was } \\
\text { performed in } \\
\text { cases of local } \\
\text { progression, } \\
\text { uncontrolled } \\
\text { bleeding, or } \\
\text { wound prob- } \\
\text { lems. } \\
\text { T3 cancer in } \\
7 \% \text {. }\end{array}$ & $\begin{array}{l}\text { OS } \\
\text { Local PFS } \\
\text { Distant PFS } \\
\text { Quality of life }\end{array}$ & $\begin{array}{l}\text { Median } \\
37.5 \text { months }\end{array}$ \\
\hline
\end{tabular}


TABLE 2. DESCRIPTION OF THE BIASES OF THE STUDIES INCLUDED. METASTATIC CANCER THERAPY - ST + SURGERY VERSUS TS RISK OF BIASES OF THE STUDIES INCLUDED

\begin{tabular}{|c|c|c|c|c|c|c|c|c|c|}
\hline Study & Random & $\begin{array}{l}\text { Allocation } \\
\text { Blinded }\end{array}$ & $\begin{array}{l}\text { Double } \\
\text { Blind }\end{array}$ & Losses & $\begin{array}{l}\text { Character- } \\
\text { istics } \\
\text { (prognostic) }\end{array}$ & Outcomes & $\begin{array}{l}\text { Sample } \\
\text { calcula- } \\
\text { tion }\end{array}$ & ITT & $\begin{array}{l}\text { Early } \\
\text { termina- } \\
\text { tion }\end{array}$ \\
\hline Badwe, 2015 & & & NA & & & & & & \\
\hline Soran, 2016 & & & NA & & & & & & \\
\hline Fitzal, 2018 & & & NA & & & & & & \\
\hline
\end{tabular}

Description of the biases of the studies included (orange = presence; blue = absence; Yellow = unclear risk of bias) ITT = analysis by intention to treat. NA = not applicable

All studies assessed the outcomes of overall survival and local progression-free survival, as well as carried out an analysis of subgroups [e.g., site and number of metastases, the status of the estrogen or progesterone receptor and status of the human epidermal growth factor receptor 2 (HER2)].

\section{Overall Survival}

Three RCTs ${ }^{(16-18)}$ compared locoregional therapy with ST $(\mathrm{N}=356)$ versus ST alone $(\mathrm{N}=358)$ and found no significant difference regarding the OS (HR: 0.93; 95\% CI 0.63 to $1.40 ; \mathrm{R}^{2}=73 \%$; evidence of very low quality, demoted due to limitations of the studies, inconsistency, and inaccuracy, Figure 1 and Tables 3 and 4.

\begin{tabular}{|c|c|c|c|c|c|}
\hline Study or Subgroup & log[Hazard Ratio] & SE & Weight & $\begin{array}{c}\text { Hazard Ratio } \\
\text { IN, Random, } 95 \% \mathrm{Cl}\end{array}$ & $\begin{array}{c}\text { Hazard Ratio } \\
\text { IV, Random, } 95 \% \mathrm{Cl}\end{array}$ \\
\hline Badwe 2015 & 0.0392 & 0.1275 & $41.0 \%$ & $1.04[0.81,1.34]$ & $\longrightarrow$ \\
\hline Fitzal 2018 & 0.3716 & 0.3432 & $20.6 \%$ & $1.45[0.74,2.84]$ & \\
\hline Soran 2016 & -0.4155 & 0.152 & $38.4 \%$ & $0.66[0.49,0.89]$ & - \\
\hline Total $(95 \% \mathrm{Cl})$ & & & $100.0 \%$ & $0.93[0.63,1.40]$ & \\
\hline \multicolumn{6}{|c|}{ 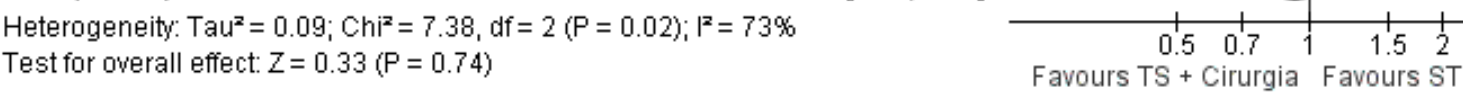 } \\
\hline
\end{tabular}

FIGURE 1. COMPARISON FOREST PLOT: 1 SYSTEMIC THERAPY WITH SURGERY VERSUS SYSTEMIC THERAPY, OUTCOME: 1.1 OVERALL SURVIVAL.

\begin{tabular}{|c|c|c|c|c|c|c|c|c|}
\hline & & & & $\begin{array}{l}\text { Hazard Ratio } \\
\text { IV, Random, 95\% Cl }\end{array}$ & & $\begin{array}{r}\text { Hazard } \\
\text { IN, Randon }\end{array}$ & $\begin{array}{l}\text { d Ratio } \\
\mathrm{m}, \mathbf{9 5} \% \mathrm{Cl}\end{array}$ & \\
\hline \multicolumn{2}{|c|}{$\frac{\text { Study or Subgroup log[Hazard Ratio] }}{\text { 1.2.1 Sobrevida lime de progressão local }}$} & \multicolumn{7}{|c|}{ 1.2.1 Sobrevida lime de progressão local } \\
\hline Badwe 2015 & -1.8326 & 0.2398 & $21.8 \%$ & $0.16[0.10,0.26]$ & $\longleftrightarrow$ & & & \\
\hline Fitzal 2018 & -0.0694 & 0.465 & $19.4 \%$ & $0.93[0.38,2.32]$ & & & & \\
\hline $\begin{array}{l}\text { Soran } 2016 \\
\text { Subtotal }(95 \% \mathrm{Cl})\end{array}$ & -0.7 & 0.816 & $\begin{array}{l}14.8 \% \\
56.1 \%\end{array}$ & $\begin{array}{r}0.50[0.10,2.46] \\
\mathbf{0 . 3 9}[0.11, \mathbf{1 . 4 5}]\end{array}$ & & & & \\
\hline \multicolumn{8}{|c|}{$\begin{array}{l}\text { Heterogeneity: } \text { Tau }^{2}=1.05 ; \mathrm{Chi}^{2}=12.17, \mathrm{df}=2(\mathrm{P}=0.002) ;\left.\right|^{2}=84 \% \\
\text { Test for overall effect: } Z=1.40(\mathrm{P}=0.16)\end{array}$} & \\
\hline \multicolumn{9}{|c|}{ 1.2.2 Sobrevida lime de progressão distante } \\
\hline Badwe 2015 & 0.3507 & 0.1396 & $22.5 \%$ & $1.42[1.08,1.87]$ & & & $\rightarrow-$ & \\
\hline $\begin{array}{l}\text { Fitzal } 2018 \\
\text { Subtotal }(95 \% \mathrm{Cl})\end{array}$ & 0.5141 & 0.2842 & $\begin{array}{l}21.4 \% \\
43.9 \%\end{array}$ & $\begin{array}{l}1.67[0.96,2.92] \\
\mathbf{1 . 4 7}[\mathbf{1 . 1 5}, \mathbf{1 . 8 7}]\end{array}$ & & & & \\
\hline \multicolumn{9}{|c|}{$\begin{array}{l}\text { Heterogeneity: } \mathrm{Tau}^{2}=0.00 ; \mathrm{Chi}^{2}=0.27, \mathrm{df}=1(\mathrm{P}=0.61) ; \mathrm{I}^{2}=0 \% \\
\text { Test for overall effect: } Z=3.05(\mathrm{P}=0.002)\end{array}$} \\
\hline Total $(95 \% \mathrm{Cl})$ & & & $100.0 \%$ & $0.72[0.25,2.04]$ & & & & \\
\hline \multicolumn{5}{|c|}{$\begin{array}{l}\text { Heterogeneity: } \operatorname{Tau}^{2}=1.24 ; \mathrm{Ch}^{2}=67.84, \mathrm{df}=4(\mathrm{P}=0.00001) ; \mathrm{I}^{2}=94 \% \\
\text { Test for overall effect: } Z=0.62(\mathrm{P}=0.54) \\
\text { Test for subqroup differences: } \mathrm{Ch}^{2}=3.77, \mathrm{df}=1(\mathrm{P}=0.05), \mathrm{I}^{2}=73.5 \%\end{array}$} & $\begin{array}{c}0.2 \\
\text { Favours TS }\end{array}$ & $\begin{array}{l}0.5 \\
\text { is cirurgia }\end{array}$ & $\begin{array}{c}2 \\
\text { Favours TS }\end{array}$ & 5 \\
\hline
\end{tabular}

FIGURE 2. COMPARISON FOREST PLOT: 1SYSTEMIC THERAPY WITH SURGERY VERSUS SYSTEMIC THERAPY, OUTCOME: 1.2 PROGRESSION-FREE SURVIVAL. 
TABLE 3. SUMMARY OF THE RESULTS FOR THE OUTCOMES OF OS, PFS, AND SUBGROUP ANALYSIS

\begin{tabular}{|c|c|c|c|}
\hline $\begin{array}{l}\text { Outcome or } \\
\text { Subgroup }\end{array}$ & $\begin{array}{l}\text { Stud- } \\
\text { ies }\end{array}$ & $\begin{array}{l}\text { Statistical } \\
\text { Method }\end{array}$ & $\begin{array}{l}\text { Estimated } \\
\text { Effect }\end{array}$ \\
\hline 1.1 Overall survival & 3 & $\begin{array}{l}\text { Hazard Ratio (IV, } \\
\text { Random, 95\% CI) }\end{array}$ & $0.93[0.63,1.40]$ \\
\hline $\begin{array}{l}1.2 \text { Progres- } \\
\text { sion-free survival }\end{array}$ & 3 & $\begin{array}{l}\text { Hazard Ratio (IV, } \\
\text { Random, 95\% Cl) }\end{array}$ & $0.72[0.25,2.04]$ \\
\hline $\begin{array}{l}1.2 .1 \text { Local } \\
\text { progression-free } \\
\text { survival }\end{array}$ & 3 & $\begin{array}{l}\text { Hazard Ratio (IV, } \\
\text { Random, 95\% CI) }\end{array}$ & $0.39[0.11,1.45]$ \\
\hline $\begin{array}{l}1.3 \text { Overall sur- } \\
\text { vival - } \\
\text { HER2 status }\end{array}$ & 3 & $\begin{array}{l}\text { Hazard Ratio (IV, } \\
\text { Random, 95\% Cl) }\end{array}$ & $0.94[0.70,1.24]$ \\
\hline $\begin{array}{l}\text { 1.3.1 HER2-pos- } \\
\text { itive }\end{array}$ & 3 & $\begin{array}{l}\text { Hazard Ratio (IV, } \\
\text { Random, 95\% Cl) }\end{array}$ & $0.91[0.64,1.30]$ \\
\hline $\begin{array}{l}\text { 1.3.2 HER2-neg- } \\
\text { ative }\end{array}$ & 3 & $\begin{array}{l}\text { Hazard Ratio (IV, } \\
\text { Random, 95\% Cl) }\end{array}$ & $0.99[0.60,1.62]$ \\
\hline $\begin{array}{l}1.4 \text { Overall sur- } \\
\text { vival - } \\
\text { SR Status }\end{array}$ & 3 & $\begin{array}{l}\text { Hazard Ratio (IV, } \\
\text { Random, 95\% CI) }\end{array}$ & $0.97[0.73,1.29]$ \\
\hline 1.4.1 SR-positive & 3 & $\begin{array}{l}\text { Hazard Ratio (IV, } \\
\text { Random, 95\% Cl) }\end{array}$ & $1.01[0.59,1.72]$ \\
\hline 1.4.2 SR-negative & 3 & $\begin{array}{l}\text { Hazard Ratio (IV, } \\
\text { Random, } 95 \% \mathrm{Cl})\end{array}$ & $0.98[0.72,1.33]$ \\
\hline $\begin{array}{l}1.5 \text { Overall } \\
\text { Survival - Bone } \\
\text { metastasis alone }\end{array}$ & 3 & $\begin{array}{l}\text { Hazard Ratio (IV, } \\
\text { Random, } 95 \% \mathrm{CI})\end{array}$ & $0.97[0.58,1.62]$ \\
\hline $\begin{array}{l}1.6 \text { Overall Sur- } \\
\text { vival - Number of } \\
\text { metastases }\end{array}$ & 1 & $\begin{array}{l}\text { Hazard Ratio (IV, } \\
\text { Random, } 95 \% \mathrm{CI} \text { ) }\end{array}$ & $1.02[0.79,1.32]$ \\
\hline $1.6 .1 \leq 3$ & 1 & $\begin{array}{l}\text { Hazard Ratio (IV, } \\
\text { Random, 95\% CI) }\end{array}$ & $1.16[0.69,1.95]$ \\
\hline $1.6 .2>3$ & 1 & $\begin{array}{l}\text { Hazard Ratio (IV, } \\
\text { Random, 95\% CI) }\end{array}$ & $0.98[0.73,1.32]$ \\
\hline $\begin{array}{l}1.7 \text { Overall Sur- } \\
\text { vival - Molecular } \\
\text { subtypes }\end{array}$ & 1 & $\begin{array}{l}\text { Hazard Ratio (IV, } \\
\text { Random, } 95 \% \mathrm{Cl} \text { ) }\end{array}$ & $1.06[0.08,13.72]$ \\
\hline 1.7.1 Luminal A & 1 & $\begin{array}{l}\text { Hazard Ratio (IV, } \\
\text { Random, 95\% Cl) }\end{array}$ & $3.62[1.25,10.50]$ \\
\hline 1.7.2 Luminal B & 1 & $\begin{array}{l}\text { Hazard Ratio (IV, } \\
\text { Random, 95\% CI) }\end{array}$ & $0.26[0.05,1.39]$ \\
\hline
\end{tabular}

TABLE 4. QUALITY OF EVIDENCE FOR THE OUTCOMES OF OVERALL AND PROGRESSION-FREE SURVIVAL (GRADE)

\begin{tabular}{|c|c|c|c|c|}
\hline Outcomes & $\begin{array}{l}\text { Relative } \\
\text { Effect } \\
(95 \% \mathrm{Cl})\end{array}$ & $\begin{array}{l}\text { No of } \\
\text { partici- } \\
\text { pants } \\
\text { (studies) }\end{array}$ & $\begin{array}{l}\text { Certainty of } \\
\text { the evidence } \\
\text { (GRADE) }\end{array}$ & $\begin{array}{l}\text { Com- } \\
\text { ments }\end{array}$ \\
\hline $\begin{array}{l}\text { Overall } \\
\text { survival } \\
\text { (OS) up to } 2 \\
\text { years ( } 23 \text { - } \\
40 \text { months) }\end{array}$ & $\begin{array}{l}\text { HR } 0.93 \\
(0.63 \text { to } \\
1.40)\end{array}$ & $\begin{array}{l}714 \\
(3 \mathrm{RCTs})\end{array}$ & $\begin{array}{l}\oplus \bigcirc \bigcirc \bigcirc \\
\text { VERY LOW } \mathbf{a , b , c}\end{array}$ & none \\
\hline $\begin{array}{l}\text { Local } \\
\text { progres- } \\
\text { sion-free } \\
\text { survival up } \\
\text { to } 2 \text { years } \\
\text { (23-40 } \\
\text { months) }\end{array}$ & $\begin{array}{l}\text { HR } 0.39 \\
(0.11 \text { to } \\
1.45)\end{array}$ & $\begin{array}{l}714 \\
(3 \mathrm{RCTS})\end{array}$ & $\begin{array}{l}\oplus O \bigcirc \bigcirc \\
\text { VERY LOW a,b, }\end{array}$ & none \\
\hline $\begin{array}{l}\text { Distant } \\
\text { progres- } \\
\text { sion-free } \\
\text { survival up } \\
\text { to } 2 \text { years } \\
\text { (23- } 40 \\
\text { months) }\end{array}$ & $\begin{array}{l}\text { HR } 1.47 \\
(1.15 \text { to } \\
1.87)\end{array}$ & $\begin{array}{l}440 \\
(2 \mathrm{RCTs})\end{array}$ & $\begin{array}{l}\oplus \oplus \oplus \bigcirc \\
\text { MODERATE }{ }^{\mathbf{a}}\end{array}$ & none \\
\hline
\end{tabular}

$\mathrm{Cl}$ : confidence interval; HR: hazard ratio; RCT: randomized controlled trial.

GRADE Working Group grades of evidence

High quality: We are very confident that the true effect lies close to that of the estimate of the effect.

Moderate quality: We are moderately confident in the effect estimate: the true effect is likely to be close to the estimate of the effect, but there is a possibility that it is substantially different.

Low quality: Our confidence in the effect estimate is limited: the true effect may be substantially different from the estimate of the effect.

Very low quality: We have very little confidence in the effect estimate: the true effect is likely to be substantially different from the estimate of effect.

\section{Progression-Free Survival}

These three RCTs ${ }^{(16-18)}$ also evaluated local progression-free survival (PFS), including a total of 714 patients, and found no significant difference in the comparison between locoregional therapy versus ST alone (HR: 0.39; $95 \%$ CI 0.11 to $1.45 ; \mathrm{R}^{2}=84 \%$; evidence of very low quality, demoted due to limitations of the studies, inconsistency, and inaccuracy Figure 2 and Tables 3 and 4.

Two studies ${ }^{(16.18)}$ including a total of 440 patients evaluated distant progression-free survival, and the group that received breast surgery with systemic treatment had a shorter time for distant PFS than the group that received the systemic treatment alone (HR: 1.47 ; 95\% CI 1.15 to $1.87 ; \mathrm{R}^{2}=0 \%$; evidence of Moderate quality, demoted due to limitations of the studies, inconsistency, and inaccuracy, Figure 2 and Tables 3 and 4 .

\section{Overall survival - HER2 status (Subgroup Analysis)}

There was no difference in overall survival between the subgroups of HER2 positive and negative (Chi ${ }^{2}=$ $\left.0.07, \mathrm{df}=1(\mathrm{P}=0.79), \mathrm{R}^{2}=0 \%\right)$, and the results for HER2 positive and negative were consistent with the primary analysis: HER2- positive HR 0.91, 95\% CI 0.64 to 1.30, I $\mathrm{I}^{2}$ \%, 3 studies, 211 patients; HER2- negative HR 0.99, $95 \%$ CI 0.60 to $1.62, I^{2} 75 \%, 3$ studies, 490 patients; Figure 3. 


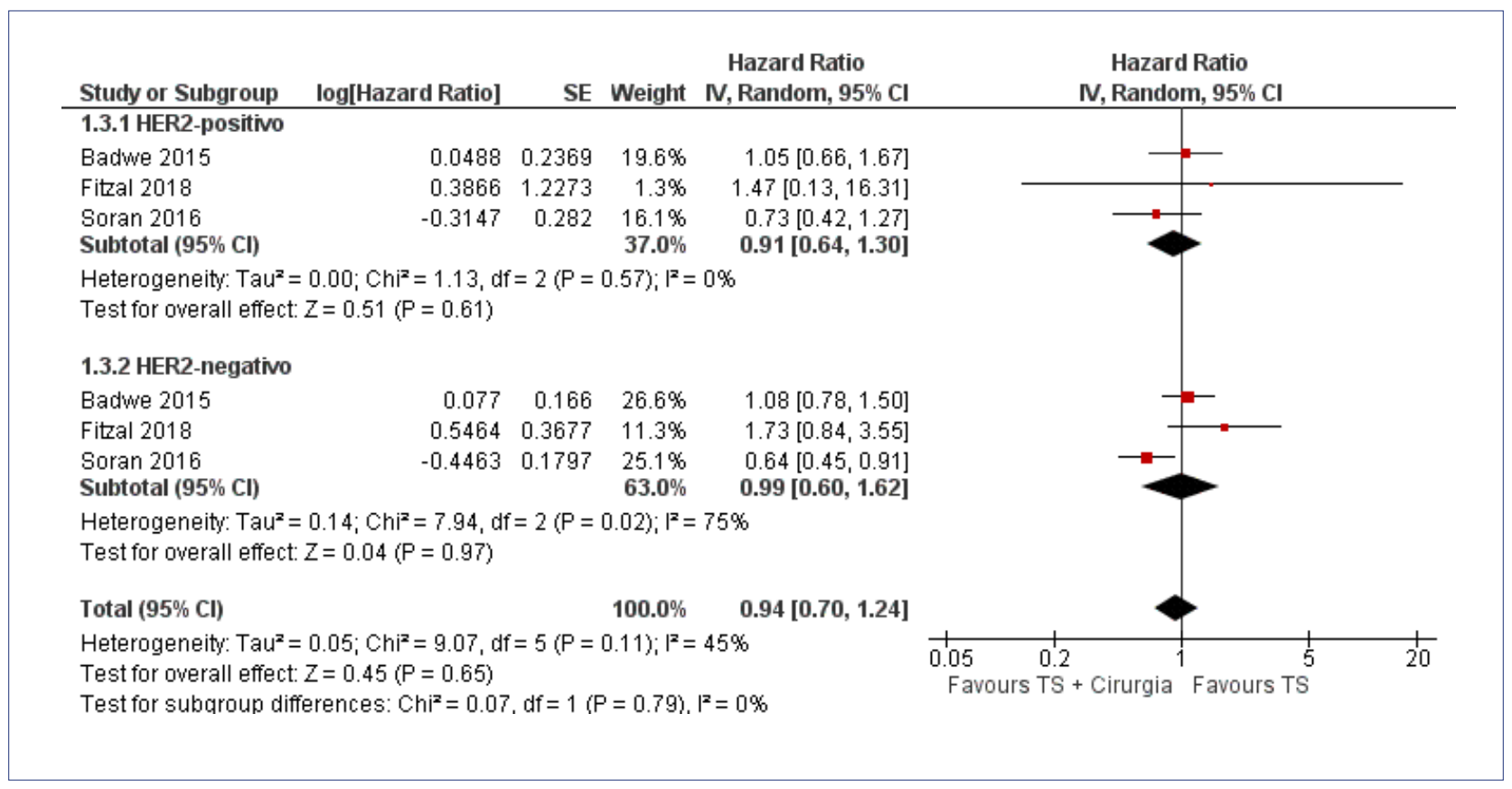

FIGURE 3. COMPARISON FOREST PLOT: 1 SYSTEMIC THERAPY WITH SURGERY VERSUS SYSTEMIC THERAPY, OUTCOME: 1.3 OVERALL SURVIVAL - HER2 STATUS.

\section{Overall survival - SR status (Subgroup Analysis)}

There was no difference in overall survival between the SR-positive and negative subgroups $\left(\mathrm{Chi}^{2}=0.01, \mathrm{df}\right.$ $\left.=1(\mathrm{P}=0.94), \mathrm{I}^{2}=0 \%\right)$, and the results for SR-positive and negative were consistent with the primary analysis: SR-positive HR 1.01, 95\% CI 0.59 to 1.72, I² 76\%, 3 studies, 496 patients; SR- negative HR 0.98, 95\% CI 0.72 to 1.33, $\mathrm{I}^{2} \mathrm{O} \%, 3$ studies, 233 patients; Figure 4.

\begin{tabular}{|c|c|c|c|c|c|c|c|}
\hline \multirow{2}{*}{$\begin{array}{l}\text { Study or Subgroup } \\
\text { 1.4.1 ER-positivo }\end{array}$} & \multirow[t]{2}{*}{ log[Hazard Ratio] } & SE & Weight & \multirow[t]{2}{*}{$\begin{array}{c}\text { Hazard Ratio } \\
\text { IV, Random, } 95 \% \mathrm{CI}\end{array}$} & \multicolumn{3}{|c|}{$\begin{array}{c}\text { Hazard Ratio } \\
\text { IV, Random, } 95 \% \mathrm{Cl}\end{array}$} \\
\hline & & & & & & & \\
\hline Badwe 2015 & 0.1044 & 0.1866 & $23.9 \%$ & $1.11[0.77,1.60]$ & & - & \\
\hline Fitzal 2018 & 0.5883 & 0.3849 & $10.4 \%$ & $1.80[0.85,3.83]$ & & & \\
\hline $\begin{array}{l}\text { Soran } 2016 \\
\text { Subtotal (95\% Cl) }\end{array}$ & -0.462 & 0.1831 & $\begin{array}{l}24.2 \% \\
58.5 \%\end{array}$ & $\begin{array}{l}0.63[0.44,0.90] \\
\mathbf{1 . 0 1}[\mathbf{0 . 5 9}, \mathbf{1 . 7 2}]\end{array}$ & & & \\
\hline \multicolumn{8}{|c|}{$\begin{array}{l}\text { Heterogeneity: } \operatorname{Tau}^{2}=0.16 ; \mathrm{Chi}^{2}=8.31, \mathrm{df}=2(\mathrm{P}=0.02) ;\left.\right|^{2}=76 \% \\
\text { Test for overall effect: } \mathrm{Z}=0.02(\mathrm{P}=0.98)\end{array}$} \\
\hline \multicolumn{8}{|l|}{ 1.4.2 ER-negativo } \\
\hline Badwe 2015 & -0.0834 & 0.1852 & $24.0 \%$ & $0.92[0.64,1.32]$ & & & \\
\hline Fitzal 2018 & -0.2771 & 0.7651 & $3.3 \%$ & $0.76[0.17,3.40]$ & & & \\
\hline $\begin{array}{l}\text { Soran } 2016 \\
\text { Subtotal }(95 \% \mathrm{Cl})\end{array}$ & 0.1989 & 0.3058 & $\begin{array}{l}14.2 \% \\
41.5 \%\end{array}$ & $\begin{array}{l}1.22[0.67,2.22] \\
\mathbf{0 . 9 8}[0.72, \mathbf{1 . 3 3}]\end{array}$ & & & \\
\hline \multicolumn{8}{|c|}{$\begin{array}{l}\text { Heterogeneity: } \mathrm{Tau}^{2}=0.00 ; \mathrm{Chi}^{2}=0.74, \mathrm{df}=2(\mathrm{P}=0.69) ;\left.\right|^{2}=0 \% \\
\text { Test for overall effect: } Z=0.12(\mathrm{P}=0.90)\end{array}$} \\
\hline Total $(95 \% \mathrm{Cl})$ & & & $100.0 \%$ & $0.97[0.73,1.29]$ & & & \\
\hline $\begin{array}{l}\text { Heterogeneity: Tau² } \\
\text { Test for overall effect } \\
\text { Test for subqroup dif }\end{array}$ & $\begin{array}{l}0.05 ; \mathrm{Ch}^{2}=9.23, \mathrm{df} \\
Z=0.19(P=0.85) \\
\text { erences: } \mathrm{Chi}^{2}=0.01\end{array}$ & $\begin{array}{l}=5(P= \\
d f=1(F\end{array}$ & $\begin{array}{l}0.10) ;\left.\right|^{2}= \\
=0.94\rangle .\end{array}$ & $\begin{array}{l}=46 \% \\
\left.\right|^{2}=0 \%\end{array}$ & \begin{tabular}{|ccc|} 
& 1 & 1 \\
0.1 & 0.2 & 0.5 \\
\multicolumn{3}{c}{ Favours } \\
TS +
\end{tabular} & $\begin{array}{c}\frac{1}{2} \\
\text { Favours TS }\end{array}$ & $\begin{array}{ll}1 & 10\end{array}$ \\
\hline
\end{tabular}

FIGURE 4. COMPARISON FOREST PLOT: 1 SYSTEMIC THERAPY WITH SURGERY VERSUS SYSTEMIC THERAPY, OUTCOME: 1.4 OVERALL SURVIVAL - SR STATUS. 


\section{Overall survival - Bone metastasis alone (Subgroup Analysis)}

For the subgroup of women with bone metastasis alone, there was no difference in overall survival (OS) when analyzing those who underwent surgery for the primary breast tumor or not: HR 0.97 (CI 95\% 0.58 to 1.62; 3 studies; 260 women; $I^{2}=51 \%$ ), Figure 5

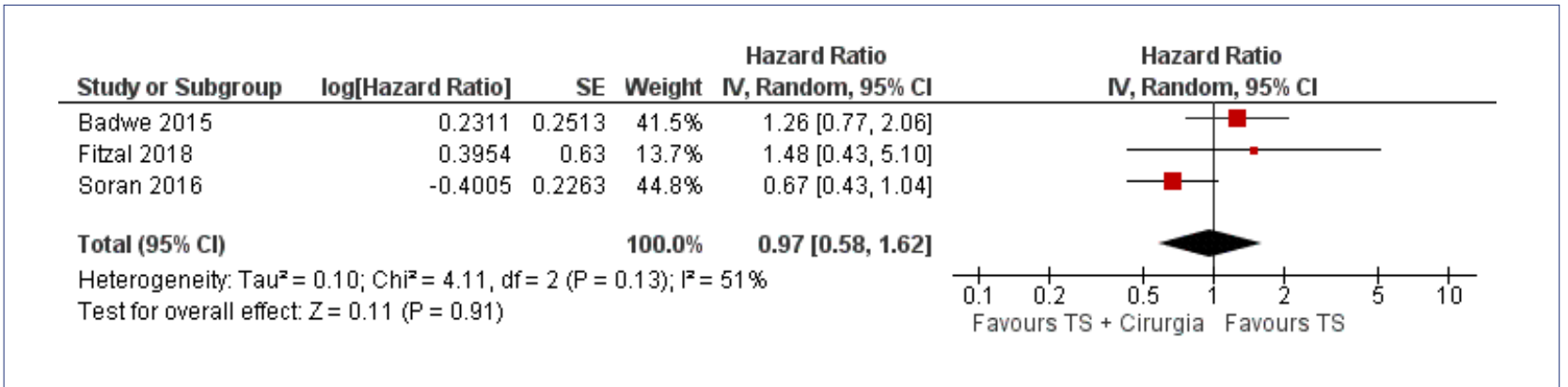

FIGURE 5. COMPARISON FOREST PLOT: 1 SYSTEMIC THERAPY WITH SURGERY VERSUS SYSTEMIC THERAPY, OUTCOME: 1.5 OVERALL SURVIVAL - BONE METASTASIS ALONE.

\section{Overall Survival - Number of metastases (Subgroup Analysis)}

Only one $\mathrm{RCT}^{(16)}$ assessed the possible relationship between the number of metastases $(\leq 3$ and $>3)$ and overall survival. There was no difference in overall survival between the $\leq 3$ and $>3$ metastases subgroups $\left(\mathrm{Chi}^{2}=0.07\right.$, $\mathrm{df}=1(\mathrm{P}=0.79))$, and the results for $\leq 3$ and $>3$ metastases were consistent with the primary analysis: $\leq 3 \mathrm{HR}$ 1.16, 95\% CI 0.69 to 1.95, 89 patients; >3 HR 0.98, 95\% CI 0.73 to 1.32, 261 patients; Figure 6.

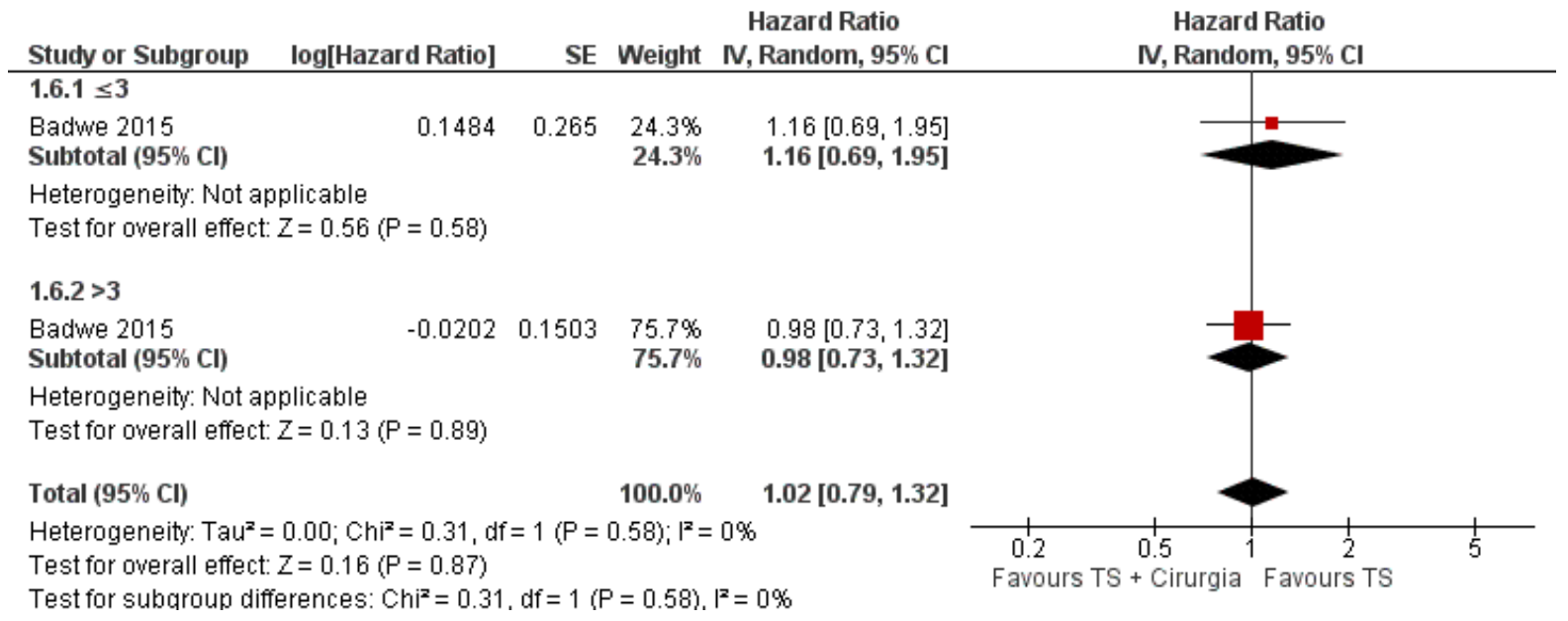

FIGURE 6. COMPARISON FOREST PLOT: 1 SYSTEMIC THERAPY WITH SURGERY VERSUS SYSTEMIC THERAPY, OUTCOME: 1.6 OVERALL SURVIVAL - NUMBER OF METASTASES.

\section{Overall survival - Molecular subtypes (Subgroup Analysis)}

One study ${ }^{(18)}$ evaluated the overall survival in the luminal A and B molecular subgroups comparing surgery with ST versus systemic therapy alone. Patients with luminal A breast cancer showed a worse performance after the initial surgery (HR 3.62, 95\% CI 1.25 to 10.50, 46 patients) and luminal B ones showed no significant difference in favor of surgery (HR 0.26, 95\% CI 0.05 to 1.39, 12 patients), Figure 7. 


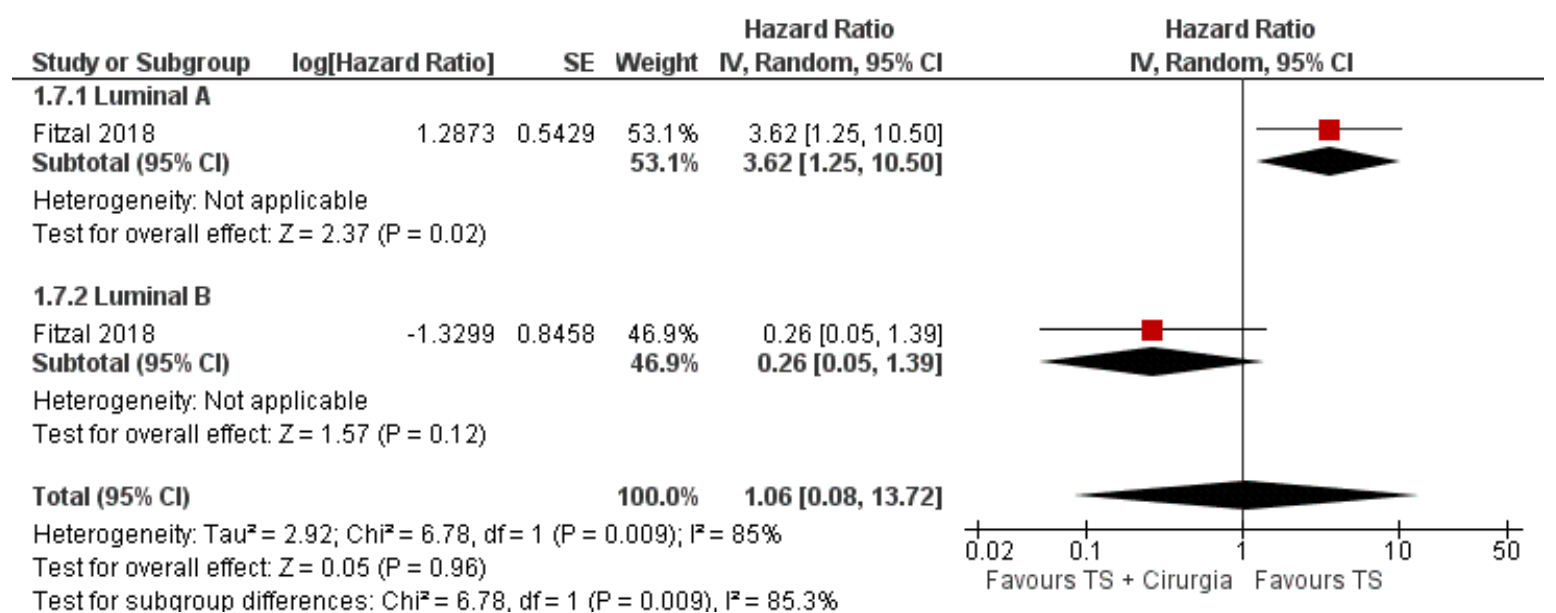

FIGURE 7. COMPARISON FOREST PLOT: 1 SYSTEMIC THERAPY WITH SURGERY VERSUS SYSTEMIC THERAPY, OUTCOME: 1.7 OVERALL SURVIVAL - MOLECULAR SUBTYPES.

\section{QUALITY OF LIFE}

One study ${ }^{(18)}$ aimed to evaluate if surgery leads to an improvement of quality of life (QOL) when compared to systemic therapy alone, based on the QOL questionnaire by the European Organization for Research and Treatment of Cancer (EORTC), i.e., the QoL questionnaire (QLQ C30, and the EORTC QLQ-BC23, a questionnaire for breast cancer patients.

Thirty-four (76\%) patients in the surgical branch and 41 (91\%) in the non-surgical branch were included in the QOL analyses.

EORTC-QLQ C30 - With time (up to 24 months of follow-up), the patients in both branches experienced clinically relevant and statistically significant improvements on the Global Health Status scale $(p=0.003)$, as well as in the emotional functioning scale. There was statistically significant worsening in the scale of dyspnea symptoms $(p=0.025)$ in both branches, but without clinical relevance.

EORTC-QLQ BR23 - In both branches, there was a statistically significant and clinically relevant improvement over time on the scale of future perspective ( $p$ $=0.009)$ and on the scale of breast symptoms $(p=$ 0.006). There was a worsening of symptoms in the scales of body image $(p=0.017)$, symptoms of systemic therapy $(p<0.001)$, and hair loss $(p<0.001)$, but these differences were not clinically relevant in both branches.

Therefore, primary tumor surgery does not improve or alter the QOL of patients with de novo breast cancer stage IV.

\section{Explanations}

a. Different prognostic characteristics among the studies, which can interfere with the results.

Fitzal, 2018 prematurely interrupted after 5 years due to poor recruitment.

b. Statistical heterogeneity.

c. $95 \%$ CI $(0.63,1.40)$, including the null effect.

d. $95 \%$ CI (0.11 to 1.45), including the null effect.

\section{SYNTHESIS OF EVIDENCE GRADE QUALITY OF EVIDENCE}

In women with metastatic breast cancer, breast surgery (mastectomy: removing the whole breast, including the nipple and areola, or Lumpectomy: removing the tumor and breast tissue around it, preserving the nipple and the areola) combined with medical treatment (such as chemotherapy and hormone therapy) compared with medical treatment alone:

- Does not improve the overall survival. The quality of the evidence is very low.

- Does not improve local progression-free survival. The quality of the evidence is very low.

- Abbreviates distant progression-free survival. Moderate quality of evidence.

- Does not improve or alter the quality of life. The quality of the evidence is very low.

\section{DISCUSSION}

Metastatic breast cancer continues to be an incurable disease despite the improvement in survival 
in recent decades, attributed mostly to advances in systemic treatment options, while the role of primary tumor resection (PTR) in this scenario remains controversial.

Based on evidence from three randomized clinical trials ${ }^{(16-18)}$, it was not possible to draw any definitive conclusions about the benefits and risks of breast surgery associated with systemic treatment for women diagnosed with metastatic breast cancer.

A RCT ${ }^{(16)}$ conducted at the Tata Memorial Hospital, in India, with 350 patients randomized to surgery versus no surgery after chemotherapy found the median overall survival was 19.2 months in the surgery group versus 20.5 months in the group without surgery. However, the patients did not receive systemic therapies according to the subtypes of breast cancer. Therapies aimed at anti-HER2 were used in only $9 \%$ of patients with the HER2-positive subtype, and very few patients with SR-positive tumors received hormone therapy.

The MF07-01 study from Turkey ${ }^{(17)}$ evaluated the prognostic effect of breast surgery as the primary treatment and observed that breast surgery can prolong the OS. However, it was not possible to confirm that surgery provides an improvement of $18 \%$ in the survival rate after three years, based on the preplanned analysis.

The phase-III randomized study ABCSG-28 (Austria) ${ }^{(18)}$ is the third to prospectively study the effectiveness of breast surgery in patients with metastases. It evaluated breast surgery for patients with de novo breast cancer stage IV, without a history of systemic therapy. The patients were allocated to surgery (standard conservative breast surgery or mastectomy, including axillary staging) with systemic therapy or systemic therapy without surgery. The patients were stratified, according to their classification, receptor status, HER2 status, site of metastases (visceral metastases versus bone alone), and first-line therapy planned. As a systemic therapy, chemotherapy, antiHER2 therapy, or anti-hormonal therapy were administered according to local standards, with schemes including modern and effective drugs. The primary outcome was the OS, and no benefit from PTR was demonstrated. In addition, there was a worsening of the results of patients with distant metastases. Due to poor recruitment, this study was prematurely interrupted after 5 years, when only 90 patients were enrolled, 45 in each branch.

A meta-analysis of these three RCTs ${ }^{(16-18)}$ showed no benefit regarding an improvement in OS, as well as in local progression-free survival, with the resection of the primary tumor (the quality of the evidence was too low); however, it indicates a shorter progression-free survival with surgery (the quality of the evidence was moderate)

We did not consider the results of subgroup analyses of the studies in this review conclusive due to the risk of false-positive results, avoiding the following question: is there indeed a significant difference in the treatment effect or it is merely a random occurrence (considering the absence of prior sample size definition and subsequent statistical power for this difference)?

\section{STUDIES IN PROGRESS}

NCT01242800. NCT00941759. NCT01242800 (ECOG2108). UMINOOOO05586 (JCOG1017)

\section{ANNEXES}

FLOWCHART . THE SELECTION OF RETRIEVED FROM THE VIRTUAL DATABASES OF SCIENTIFIC INFORMATION IS DETAILED IN THE FLOWCHART BELOW:

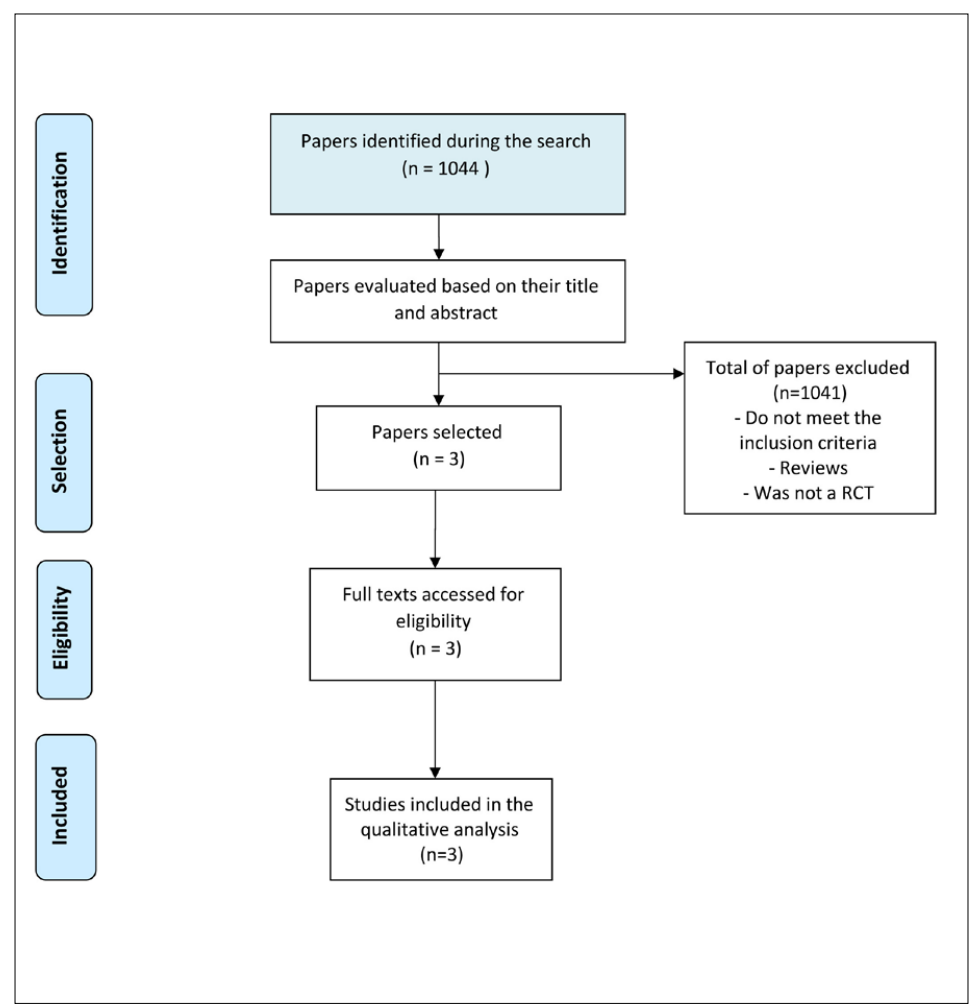




\section{REFERENCES}

1. Global Burden of Disease Cancer Collaboration. Global, Regional, and National Cancer Incidence, Mortality, Years of Life Lost, Years Lived With Disability, and Disability- Adjusted Life-years for 32 Cancer Groups, 1990 to 2015: A Systematic Analysis for the Global Burden of Disease Study. JAMA Oncology 2017;3(4):524-48.

2. INCA - Instituto Nacional de Câncer |https://www.inca.gov.br

3. Finn RS, Crown JP, Lang I, Boer K, Bondarenko IM, Kulyk SO, et al. The cyclin-dependent kinase 4/6 inhibitor palbociclib in combination with letrozole versus letrozole alone as first-line treatment of oestrogen receptor-positive, HER2-negative, advanced breast cancer (PALOMA-1/TRIO-18): a randomised phase 2 study. Lancet Oncol 2015;16:25-35. PMID: 25524798

4. Simon SD, Bines |, Werutsky G, Nunes IS, Pacheco FC, Segalla |G, et al. Characteristics and prognosis of stage I-III breast cancer subtypes in Brasil: The AMAZONA retrospective cohort study. Breast 2019;44:113-119. PMID: 30738289

5. Colozza M, E. de Azambuja de E, Personeni N, et al., Achievements in systemic therapies in the pregenomic era in metastatic breast cancer, Oncologist 12 (2007)

6. Rashid OM, Takabe K. Does removal of the primary tumor in metastatic breast cancer improve survival? J. Womens Health 23 (2) (2014) 184-188.

7. Badwe R, Hawaldar R, Nair N, et al., Locoregional treatment versus no treatment of the primary tumour in metastatic breast cancer: an open-label randomised controlled trial, Lancet Oncol. 16 (2015) 1380-1388.

8. Khan SA Stewart AK, Morrow M. Does aggressive local therapy improve survival in metastatic breast cancer? Surgery 132 (4) (2002) 620-626.

9. Babiera GV, Rao R, Feng $L$, et al. Effect of primary tumor extirpation in breast cancer patients who present with stage IV disease and an intact primary tumor, Ann. Surg. Oncol. 13 (6) (2006) 776-782.

10. Fields RC, Jeffe DB, Trinkaus $K$, et al. Surgical resection of the primary tumor is associated with increased long-term survival in patients with stage IV breast cancer after controlling for site of metastasis, Ann. Surg. Oncol. 14 (12) (2007) 3345-3351.

11. Kandace P, McGuire SE, Rodriguez A, et al. Factors associated with improved outcome after surgery in metastatic breast cancer patients, Am. J. Surg. 198 (2009) 511-515.

12. Moher D, Liberati A, TetzlaffJ, Altman DG, The PRISMA Group. Preferred Reporting Items for Systematic Reviews and Meta-Analyses: The PRISMA Statement. Disponível em: www.prisma-statement.org.

13. Review Manager (RevMan) [Computer program]. Version 5.3. Copenhagen: The Nordic Cochrane Centre, The Cochrane Collaboration, 2014.

14. Higgins JPT, Thomas J, Chandler J, Cumpston M, Li T, Page MJ, Welch VA (editors). Cochrane Handbook for Systematic Reviews of Interventions version 6.0 (updated July 2019). Cochrane, 2019. Available from www.training. cochrane.org/handbook

15. GRADEpro GDT: GRADEpro Guideline Development Tool [Software]. McMaster University, 2015 (developed by Evidence Prime, Inc.). Available from gradepro.org.

16. Badwe R, Hawaldar R, Nair N, Kaushik R, Parmar V, Siddique S, et al. Locoregional treatment versus no treatment of the primary tumour in metastatic breast cancer: an open-label randomised controlled trial. Lancet Oncol 2015;16:1380-8. PMID: 26363985

17. Soran A, Ozmen V, Ozbas S, Karanlik H, Muslumanoglu M, Igci A, et al. Randomized Trial Comparing Resection of Primary Tumor with No Surgery in Stage IV Breast Cancer at Presentation: Protocol MF07-01. Ann Surg Oncol 2018;25:3141-3149. PMID: 29777404

18. Fitzal F, Bjelic-Radisic V, Knauer M, Steger G, Hubalek M, Balic M, Singer $C$, et al. Impact of Breast Surgery in Primary Metastasized Breast Cancer: Outcomes of the Prospective Randomized Phase III ABCSG-28 POSYTIVE Trial. Ann Surg 2019;269:1163-1169. PMID: 31082916 